\title{
COMPACTIFICATIONS OF THE RAY WITH THE ARC AS REMAINDER ADMIT NO $n$-MEAN
}

\author{
M. M. AWARTANI AND DAVID W. HENDERSON
}

(Communicated by James E. West)

\begin{abstract}
An $n$-mean on $X$ is a function $F: X^{n} \rightarrow X$ which is idempotent and symmetric. In $1970 \mathrm{P}$. Bacon proved that the $\sin (1 / x)$ continuum admits no 2-mean. In this paper, it is proved that if $X$ is any metric space which contains an open line one of whose boundary components in $X$ is an arc, then $X$ admits no $n$-mean, $n \geq 2$.
\end{abstract}

\section{INTRODUCTION}

An $n$-mean $(n \geq 2)$ is a continuous function $m: X^{n} \rightarrow X$ satisfying the conditions:

(i) $m$ is idempotent: $m(x, \ldots, x)=x$ for each $x \in X$,

(ii) $m$ is symmetric: $m\left(x_{1}, \ldots, x_{n}\right)=m\left(x_{\sigma(1)}, \ldots, x_{\sigma(n)}\right)$ for each permutation $\sigma$ on $\{1, \ldots, n\}$.

The problem of determining those spaces which admit an $n$-mean was first considered by G. Auman and Caratheodory (see, e.g., [1]), and later by B. Eckmann, T. Ganea, and P. J. Hilton (see [4]). In [4] it is proved that if $X$ is a compactum with the homotopy type of a compact polyhedron, then $X$ admits an $n$-mean $(n \geq 2)$ only if $X$ is contractible.

Means on nonlocally connected continua were first studied by A. D. Wallace [10], who conjectured that the $\sin (1 / x)$ continuum admits no 2-mean. In $1968 \mathrm{~K}$. Sigmon [9] showed that the $\sin (1 / x)$ continuum admits no distributive mean, and in 1970 P. Bacon [3] proved Wallace's conjecture. The results that K. Sigmon and P. Bacon obtained in their deep investigation of means included some strong necessary conditions for the existence of a mean. Their approach used tools of various homology and cohomology theories. For example, K. Sigmon used Alexander Cohomology to show [8] that if $X$ is a continuum which admits an $n$-mean, then $H^{p}\left(X, Z_{n}\right)=0, p \geq 1$ (in Alexander Cohomology); $X$ cannot separate $\mathbb{R}^{k}, k \geq 2$; and $X$ is unicoherent.

A compactification of the ray $J=(0,1]$ is a compact space $X$ in which $J$ is densely embedded. $X-J$ is called the remainder of the compactification.

Received by the editors December 7, 1992.

1991 Mathematics Subject Classification. Primary 54F15; Secondary 54D35.

Key words and phrases. Compactification of the ray, $n$-mean, mean, essential maps.

This research was done while the first author was a visitor at Cornell University during the summer of 1991.

(C)1995 American Mathematical Society 
The purpose of this paper is to establish the following generalization of Bacon's result through essentially geometric methods.

Theorem 1.1. If $X$ is any compactification of the rav with the arc as remainder, then $X$ admits no $n$-mean, $n \geq 2$.

As a corollary to the above theorem we obtain the following:

Theorem 1.2. If $X$ is any metric space which contains an open line one of whose boundary components in $X$ is an arc, then $X$ admits no n-mean, $n \geq 2$.

Notice that the $\sin (1 / x)$ continuum is the simplest member within the class of compactifications of the ray with the arc as remainder. This family was shown [2] to contain uncountably many continua no one of which maps onto any other.

\section{MEANS AND ESSENTIAL MAPS}

Notation and Definition 2.1. Throughout this paper the following notation will be adopted: $X^{n}$ denotes the Cartesian product of $n$ copies of $X$ with the product topology. If $f: X \rightarrow Y$ is a map, then $f^{n}: X^{n} \rightarrow Y^{n}$ is defined as: $f^{n}\left(x_{1}, \ldots, x_{n}\right)=\left(f\left(x_{1}\right), \ldots, f\left(x_{n}\right)\right)$. $\pi$ denotes the projection of $\mathbb{R}^{2}$ onto the $y$-axis. Finally, if $p$ and $q$ are two points of some embedding $J$ of the ray, then $[p, q]_{J}$ denotes the arc in $J$ whose endpoints are $p$ and $q$. The function $f: X \rightarrow(Y, B), B \subseteq Y$, is inessential if there exists a map $g: X \rightarrow B$ such that $g\left|f^{-1}(B)=f\right| f^{-1}(B)$. A map is essential if it is not inessential.

Lemma 2.2. If $X$ and $Y$ are two arcs and $f: X \rightarrow Y$ is a map which maps the boundary of $X$ onto the boundary of $Y$, then

(i) $f: X \rightarrow(Y$, bd $Y)$ is essential, and

(ii) $f^{n}: X^{n} \rightarrow\left(Y^{n}\right.$, bd $\left.Y^{n}\right)$ is essential.

Proof. (i) If there were a map $g: X \rightarrow$ bd $Y$ such that $g \mid f^{-1}($ bd $Y)=f$, then $g(x)$ would be a disconnected set with two points.

(ii) The map $f$ is clearly homotopic $(\bmod b d X)$ to a homeomorphism and thus as a map of pairs $f^{n}:\left(X^{n}, \mathrm{bd} X^{n}\right) \rightarrow\left(Y^{n}, \mathrm{bd} Y^{n}\right)$ is homotopic to a homeomorphism $k:\left(X^{n}, \mathrm{bd} X^{n}\right) \rightarrow\left(Y^{n}, \mathrm{bd} Y^{n}\right)$. If there were a map $k: X^{n} \rightarrow$ bd $Y^{n}$ such that $k\left(f^{n}\right)^{-1}\left(\mathrm{bd} Y^{n}\right)=f^{n}$, then $k \mid \mathrm{bd} X^{n}$ would be homotopic to $h \mid \operatorname{bd} X^{n}$ and thus $h \mid \operatorname{bd} X^{n} \rightarrow \operatorname{bd} Y^{n}$ would be homotopically trivial (in bd $Y^{n}$ ), which is impossible.

Note. Lemma 2.2 can be extended to the case when $X$ is a general metric continuum, but the lemma is false if $Y$ is allowed to be $I^{2}$. Specifically, in [7] there is constructed a compactum $X$ with $\operatorname{dim} X=2$ but $\operatorname{dim} X^{2}=3$. It follows from [5], Theorems VI2 and VI4 and the remarks on pages 78-79, that $X$ has an essential mapping onto $\left(I^{2}\right.$, bd $\left.I^{2}\right)$ but $X^{2}$ has no essential mapping onto $\left(I^{4}=I^{2} \times I^{2}\right.$, bd $\left.I^{4}\right)$.

Lemma 2.3. Let $X$ be a continuum, and let $f: X \rightarrow\left(I^{n}\right.$, bd $\left.I^{n}\right)$ be an essential map. Let $K$ be any continuum which is the closure of an open set in $I^{n}$ and which is the intersection of a sequence of open subsets of $I^{n}: U_{1} \supset U_{2} \supset U_{3} \supset \cdots$, such that each $\mathrm{cl} U_{i}$ is homeomorphic to an $n$-ball. Then some continuum in $X$ maps onto $K$. 
Proof. We shall show that if no component of $X$ maps onto $K$, then $f: X \rightarrow$ $\left(I^{n}\right.$, bd $\left.I^{n}\right)$ is inessential. Let $C_{1}, C_{2}, \ldots$ be the components of $f^{-1}(K)$. For each $i$ pick integer $j(i)$ and open set $V_{i}$ so that

(a) $V_{i}$ is a component of $f^{-1}\left(U_{j(i)}\right)$,

(b) $C_{i} \subset V_{i}$,

(c) $V_{i} \cap V_{j}=\varnothing, i \neq j$, and

(d) $f\left(\mathrm{cl} V_{i}\right)$ is not onto $K$.

We now define a new map $\hat{f}: X \rightarrow I^{n}$ such that $\hat{f}\left|X-\bigcup\left\{V_{i}\right\}=f\right| X-\bigcup\left\{V_{i}\right\}$ and $\hat{f} \mid \mathrm{cl} V_{i}$ is defined as follows: Let $p_{i} \in\left(K-f\left(\operatorname{cl} V_{i}\right)\right) \cap \operatorname{int} I^{n}$ and let $r_{i}$ be a retract of $\operatorname{cl} U_{j(i)}-p_{i}$ onto bd $V_{j(i)}$. Define $\hat{f}\left|\operatorname{cl} V_{i}=r_{i} \circ f\right| \operatorname{cl} V_{i}$. Note that this makes sense since $f\left(\operatorname{bd} V_{i}\right) \subset \operatorname{bd} V_{j(i)}$. Note also that $\hat{f} \mid f^{-1}\left(\operatorname{bd} I^{n}\right)=$ $f \mid f^{-1}\left(\mathrm{bd} I^{n}\right)$ since $\mathrm{cl} U_{j(i)} \cap \mathrm{bd} I^{2} \subset$ bd $U_{j(i)}$.

Now $\hat{f}(X) \subset I^{n}-\left(K \cap\right.$ int $\left.I^{n}\right)$. So pick a point $p \in K_{n} \cap$ int $I^{n}$ and let $r$ be a retract of $I^{n}-\{p\}$ onto bd $I^{n}$. Then $g=r \circ \hat{f}: X \rightarrow$ bd $I^{n}$ is such that $g\left|f^{-1}\left(\mathrm{bd} I^{n}\right)=f\right| f^{-1}\left(\mathrm{bd} I^{n}\right)$, which contradicts our hypotheses.

Lemma 2.4. If $G$ and $H$ are open sets covering $I^{n}$ such that $\{(x, x, \ldots, x) \mid 0 \leq$ $\left.x \leq \frac{1}{2}\right\} \subset G$ and $\left\{(x, x, \ldots, x) \mid \frac{1}{2} \leq x \leq 1\right\} \subset H$, then either

(i) the component of $G$ containing $(0,0, \ldots, 0)$ intersects $B_{1}$ [ $\equiv$ the union of the $(n-1)$-faces of bd $I^{n}$ which contain $\left.(1,1, \ldots, 1)\right]$, or

(ii) the component of $H$ containing $(1,1, \ldots, 1)$ intersects $B_{0}[\equiv$ the union of the $(n-1)$-faces of bd $I^{n}$ which contain $\left.(0,0, \ldots, 0)\right]$.

Proof. Let $\widetilde{G}[\widetilde{H}]$ be the component of $G[H]$ containing $(0,0, \ldots, 0)$ $[(1,1, \ldots, 1)]$. bd $\widetilde{G}$ separates $(0,0, \ldots, 0)$ from $(1,1, \ldots, 1)$ and thus one component $D$ of bd $\widetilde{G}$ separates $I^{n}$ into open sets $U(\supset(0,0, \ldots, 0))$ and

$V(\supset(1,1, \ldots, 1))$. Note that, since $\widetilde{H} \cap G \neq \varnothing$, it must be true that $\tilde{H} \cap D \neq \varnothing$ and thus $D \subset \widetilde{H}$. Hence either $D \cap B_{0} \neq \varnothing$ or $D \cap B_{0}=\varnothing$. If $D \cap B_{0} \neq \varnothing$, then conclusion (ii) holds. If $D \cap B_{0}=\varnothing$, then conclusion (i) holds.

Lemma 2.5. Let $A=A_{1} \cup A_{2}$ where $A_{1}$ and $A_{2}$ are arcs in the plane such that $A_{1} \cap A_{2}=\{p\}$ is an endpoint of both $A_{1}$ and $A_{2}$ and such that $\pi \mid A_{1}$ and $\pi \mid A_{2}$ are both homeomorphisms onto $I$. If $K$ is any subcontinuum of $I^{n}$ which intersects each $(n-1)$-face of bd $I^{n}$ containing $(\pi(p), \pi(p), \ldots, \pi(p))$, then $\left(\pi^{n}\right)^{-1}(K)$ is a subcontinuum of $A^{n}$.

Proof. For each of the $2^{n} n$-cells $A^{n}=A_{i_{1}} \times A_{i_{2}} \times \cdots \times A_{i_{n}}, i_{j}=1$ or $2, \pi \mid A^{n}$ is a homeomorphism and thus $\left(\pi^{n}\right)^{-1}(K) \cap A^{n}$ is a continuum. Let

$$
K_{1}=\left(\pi^{n}\right)^{-1}(K) \cap\left(A_{1}^{n}\right) \quad \text { and } \quad K_{2}=\left(\pi^{n}\right)^{-1}(K) \cap\left(A_{2} \times A_{1}^{n-1}\right) .
$$

Then $A_{1}^{n} \cap\left(A_{2} \times A_{1}^{n-1}\right)=\{p\} \times A_{1}^{n-1}$ and by hypothesis $K$ intersects $\pi\left(\{p\} \times A_{1}^{n-1}\right)=\pi(p) \times A_{1}^{n-1}$. Thus $K_{1} \cup K_{2}$ is connected. Continuing likewise we see that $\left(\pi^{n}\right)^{-1}(K)$ is connected.

\section{EMBEDDING COMPACTIFICATIONS OF THE RAY}

Theorem 3.1 [6]. If $K$ is a continuum which is embeddable in $\mathbb{R}^{n}$, then any compactification of a ray with $K$ as remainder is embeddable in $\mathbb{R}^{n+1}$.

The proof of the above theorem actually gives the embedding in $\mathbb{R}^{n} \times[0,1]$ as the closure of the graph of a piecewise linear function $g:(0,1] \rightarrow \mathbb{R}^{n}$. 
In the case when $K$ is an arc, the proof of the above theorem can be slightly modified to give the following:

Lemma 3.2. Let $S$ be a compactification of the ray with the arc as remainder. Then $S$ can be embedded in the plane as the closure of the graph of a piecewise linear function $g:(0,1] \rightarrow[0,1]$ such that for every $y \in[0,1], f^{-1}(y)$ is a sequence in $(0,1]$ converging to 0.

Corollary 3.3. Let $S$ be a compactification of the ray with the arc as remainder. Then the following hold:

(a) There exists a sequence $\left\{q_{i}\right\}$ in $J \cap \pi^{-1}(1)$ such that $\pi \mid\left[q_{2 i-1}, q_{2 i}\right]_{J}$ is a surjection onto $I$ and where $\pi^{-1}(1) \cap\left[q_{2 i-1}, q_{2 i}\right]_{J}=\left\{q_{2 i-1}, q_{2 i}\right\}$.

(b) There exists a sequence $\left\{p_{i}\right\}$ in $J \cap \pi^{-1}(p)$ such that $\pi \mid\left[p_{2 i-1}, p_{2 i}\right]_{J}$ is a surjection onto $I$ and where $\pi^{-1}(p) \cap\left[p_{2 i-1}, p_{2 i}\right]_{J}=\left\{p_{2 i-1}, p_{2 i}\right\}$.

\section{Proofs of MAIN THEOREMS}

Proof of Theorem 2.2. Let $S$ be a compactification of the ray with the arc as remainder, and suppose that $m: S^{n} \rightarrow S$ is an $n$-mean, for some $n \geq 2$. Let $G=\left\{(x, y) \in S \mid y>\frac{1}{3}\right\}, H=\left\{(x, y) \in S \mid y<\frac{2}{3}\right\}$, and let $\left\{G^{\prime}, H^{\prime}\right\}$ be an open cover of $S$, such that $\mathrm{cl} G^{\prime} \subset G$ and $\operatorname{cl} H^{\prime} \subset H$. Hence $\left\{m^{-1}\left(G^{\prime}\right), m^{-1}\left(H^{\prime}\right)\right\}$ is an open cover of $S^{n}$. By Lemma 2.4, two cases arise:

Case (i): The component of $(1,1, \ldots, 1)$ in $m^{-1}\left(H^{\prime}\right) \cap I^{n}$ intersects $B_{0}$. Let $L$ denote the closure of this component. By symmetry of $m$, it follows that $L$ intersects each $(n-1)$-face of $B_{0}$. Let $\left\{q_{i}\right\}$ be the sequence guaranteed by part (a) of Lemma 3.3, and let $i$ be chosen large enough so that $\left(\pi^{n}\right)^{-1}(L) \cap$ $\left[q_{i}, q_{i+1}\right]_{J}^{n} \subseteq m^{-1}(H)$. Let $p \in\left[q_{i}, q_{i+1}\right]_{J} \cap \pi^{-1}(0)$.

Define a function $\pi^{\prime}:\left[q_{i}, q_{i+1}\right]_{J} \rightarrow\left[q_{i}, p\right]_{\mathbb{R}^{2}} \cup\left[p, q_{i+1}\right]_{\mathbb{R}^{2}}$, where $A_{1}=$ $\left[q_{i}, p\right]_{\mathbb{R}^{2}}$ and $A_{2}=\left[p, q_{i+1}\right]_{\mathbb{R}^{2}}$ denote the straight line segments joining $q_{i}$ to $p$ and $q_{i+1}$ to $p$, respectively. Since $\pi \mid\left[g_{i}, p\right]_{\mathbb{R}^{2}}$ and $\pi \mid\left[g_{i+1}, p\right]_{\mathbb{R}^{2}}$ are both homeomorphisms onto $I$, it follows from Lemma 2.5 that $L^{\prime}=\left(\pi^{n}\right)^{-1}(L) \cap$ $\left(A_{1} \cup A_{2}\right)^{n}$ is a continuum containing $\left(q_{i}, \ldots, q_{i}\right)$ and $\left(q_{i+1}, \ldots, q_{i+1}\right)$. Since $\pi^{\prime n}$ is an essential map, it follows from Lemma 2.3 that $\left(\pi^{\prime n}\right)^{-1}\left(L^{\prime}\right)$ contains a component $L^{\prime \prime}$ such that $\pi^{\prime n}\left(L^{\prime \prime}\right)=L^{\prime}$. Notice that $\pi^{n}\left(L^{\prime \prime}\right)=\pi^{n} \circ \pi^{\prime n}\left(L^{\prime \prime}\right)=$ $L$. Hence $L^{\prime \prime} \subseteq\left(\pi^{n}\right)^{-1}(L) \cap\left[q_{i}, q_{i+1}\right]_{J}^{n} \subseteq m^{-1}(H)$. Since $L^{\prime \prime}$ contains the points $\left(q_{i}, \ldots, q_{i}\right)$ and $\left(q_{i+1}, \ldots, q_{i+1}\right)$, it follows that both of them belong to the same component of $m^{-1}(H)$. This contradicts the fact that their images $m\left(q_{i}, \ldots, q_{i}\right)=q_{0}$ and $m\left(q_{i+1}, \ldots, q_{i+1}\right)=q_{i+1}$ belong to different components of $H$. Hence the original assumption that $m^{-1}(H) \cap I^{n}$ intersects $B_{0}$ is false.

Case (ii): The component of $(0,0, \ldots, 0)$ in $m^{-1}(G)$ intersects $B_{1}$. Using an argument similar to the one above but replacing the sequence $\left\{q_{i}\right\}$ with the sequence $\left\{p_{i}\right\}$ from part (b) of Lemma 3.3, we conclude that for suffcient large $i,\left(p_{i}, \ldots, p_{i}\right)$ and $\left(p_{i+1}, \ldots, p_{i+1}\right)$ belong to the same component of $m^{-1}(G)$, contradicting the fact that their images $m\left(p_{i}, \ldots, p_{i}\right)=p_{i}$ and $m\left(p_{i+1}, \ldots, p_{i+1}\right)=p_{i+1}$ belong to different components of $G$. Hence the assumption that the component of $(0, \ldots, 0)$ in $m^{-1}(G)$ intersects $B_{1}$ is also false. But the conclusions of the above two cases are jointly inconsistent with Lemma 2.4. This completes the proof of Theorem 1.1. 
Proof of Theorem 1.2. Let $X$ be a metric space which contains an open line $L$ one of whose boundary components in $X$ is an arc $I$. Then $L$ contains a half line (ray), $J$, such that $\mathrm{cl} J-J$ is $I$ or a subarc of $I$. Suppose that $m: X^{n} \rightarrow X$ is an $n$-mean. Since $m$ is idempotent, it follows that there exists a subray $J^{\prime} \subset J$ such that $\operatorname{cl} J^{\prime}-J^{\prime}=I$ and $\operatorname{cl} J^{\prime} \subseteq m\left(\operatorname{cl} J^{\prime n}\right) \subseteq \operatorname{cl} J$. Let $a, a^{\prime}$ denote the endpoints of the rays $J$ and $J^{\prime}$ respectively. Define a function $g: \operatorname{cl} J \rightarrow \operatorname{cl} J^{\prime}$ as follows: $g \mid \operatorname{cl} J^{\prime}$ is the identity and $g(x)=a^{\prime}$ for each $x \in\left[a^{\prime}, a\right] \subset J$. Then the map $m^{\prime}: \operatorname{cl} J^{\prime n} \rightarrow \operatorname{cl} J^{\prime}$ given by $m^{\prime}=g \circ m$ is clearly an $n$-mean on $\mathrm{cl} J^{\prime}$, which contradicts Theorem 1.1 , since $\operatorname{cl} J^{\prime}$ is a compactification of the ray with the arc as remainder. This completes the proof of Theorem 1.2.

\section{REFERENCES}

1. G. Auman, Über Raüme mit Mittlebildungen, Math. Ann. 119 (1943), 210-215.

2. M. Awartani, An uncountable collection of mutually incomparable chainable continua, Proc. Amer. Math. Soc. 118 (1993), 239-245.

3. P. Bacon, An acyclic continuum that admits no mean, Fund. Math. 67 (1970), 11-13.

4. B. Eckmann, T. Ganea, and P. J. Hilton, Generalized means, Studies in Mathematical Analysis and Related Topics, Stanford Univ. Press, Stanford, CA, 1962.

5. W. Hurewicz and H. Wallman, Dimension theory, Princeton Univ. Press, Princeton, NJ, 1941.

6. S. Nadler, Embedding certain compactifications of half ray, Fund. Math. 70 (1973), 217-225.

7. L. Pontryagin, Sur une hypothése fondamentale de la théorie de la dimension, Comptes Rendus 190 (1920), 1105-1107.

8. K. Sigmon, On the existence of mean on certain continua, Fund. Math. 63 (1969), 311-319.

9. __ Acyclicity of compact means, Michigan Math. J. 16 (1969), 111-115.

10. A. D. Wallace, Acyclicity of compact connected semigroups, Fund. Math. 50 (1961), 99-105.

Department of Mathematics, Birzeit University, Birzeit, West Bank

E-mail address: marwan@psms.birzeit.edu

Department of Mathematics, Cornell University, Ithaca, New York 14853-7901

E-mail address: dwh@math.cornell.edu 